\title{
Squamous cell carcinomas and adenocarcinomas of the esophagus: One treatment does not rule them all
}

\author{
Kiran H. Lagisetty, David G. Beer, and Andrew C. Chang
}

\footnotetext{
From the Section of Thoracic Surgery, Department of Surgery, University of Michigan Medical School, Ann Arbor, Mich.

Received for publication April 10, 2017; accepted for publication April 18, 2017; available ahead of print June 27, 2017

Address for reprints: Andrew C. Chang, MD, 1500 East Medical Center Dr, Ann Arbor, MI 48109 (E-mail: andrwchg@med.umich.edu).

J Thorac Cardiovasc Surg 2017;154:1446-7

$0022-5223 / \$ 36.00$

Copyright (c) 2017 by The American Association for Thoracic Surgery

http://dx.doi.org/10.1016/j.jtcvs.2017.04.074
}

Feature Editor's Note-Clinicians have long realized that squamous cell carcinomas and adenocarcinomas of the esophagus behave differently, particularly in their response to chemoradiotherapy. Yet these 2 histologic subtypes of esophageal cancer have traditionally been considered a single entity, with similar treatment recommendations. Importantly, many of the clinical trials that become the basis for our standard of care in esophageal cancer include tumors of both histologies, often resulting in a tempered interpretation of trial data. In a recent issue of Nature (reference 1 in this Feature Expert Opinion), the authors of The Cancer Genome Atlas (TCGA) provide a comprehensive understanding of the intrinsic differences in the genomic underpinnings between these two tumors. This landmark paper provides definitive molecular evidence that esophageal squamous cell carcinomas and esophageal adenocarcinomas are 2 distinct tumors. In fact, several analyses demonstrate that each of these tumors is more similar to a nonesophageal cancer than they are similar to each other. We are pleased to present a Feature Expert Opinion on this article by Lagisetty, Beer, and Chang, a group with great experience in this field. You will find below a thoughtful, concise, and relevant interpretation of the TCGA's multiplatform genomic esophageal cancer data, with important implications for clinical research and clinical trial design that can help us refine our efforts in the battle against this aggressive malignancy.

\section{Bryan Burt, MD}

Historically, squamous cell carcinoma (ESCC) and adenocarcinoma (EAC) of the esophagus have been considered a single entity with similar treatment recommendations in terms of operative resection and oncology therapy. Recent evidence from The Cancer Genome Atlas Research Network (TCGA) suggests that in fact this might not be true. ${ }^{1}$ Using a combination of high-throughput sequencing and molecular analysis methods, integrative cluster analysis identified distinctive genomic and molecular differences between tumors of squamous cell and adenocarcinoma histology. Furthermore, these investigators confirmed that the EAC molecular phenotype is

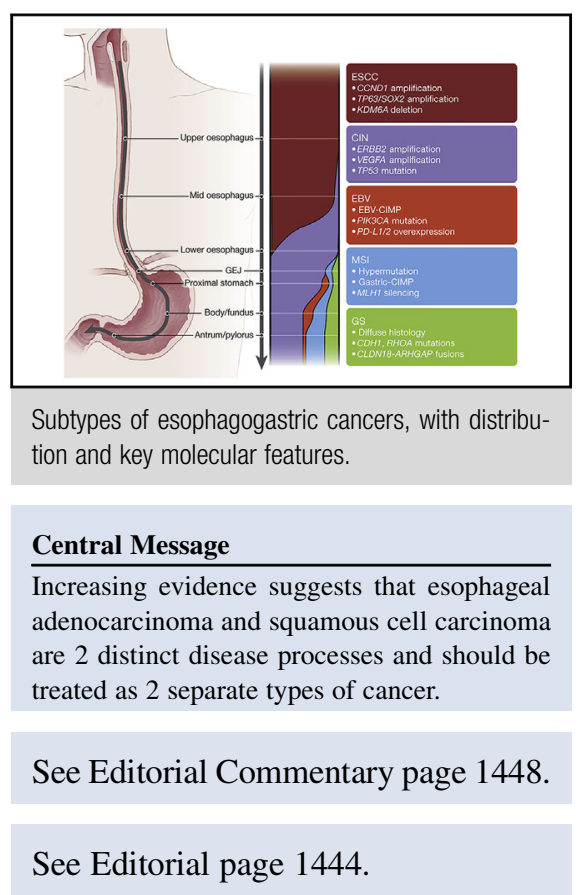

more like esophagogastric junction carcinomas and colon carcinomas than squamous cell carcinomas. Just as the TNM criteria for staging ESCC and EAC are distinctly different, these cancers also should be treated independently when designing clinical trials for esophageal cancer.

Since its inception, the TCGA Research Network, jointly funded by the National Cancer Institute and the National Human Genome Research Institute of the National Institutes of Health, has collected specimens from more than 11,000 patients with 33 different types of cancer. These tissues have been analyzed for genomic alterations on a large scale, and findings from these studies have provided the molecular basis for developing targeted therapies in a variety of cancer types (https://cancergenome.nih.gov/publications).

Earlier this year, the TCGA Research Network reported their analysis of an international cohort of fresh frozen tumor samples obtained from 164 patients with esophageal cancer, 359 patients with gastric adenocarcinoma, and 36 patients with esophagogastric junction carcinoma previously untreated by chemotherapy or radiation. Wholeexome sequencing, single-polymorphism array profiling, DNA methylation profiling, and messenger RNA and microRNA sequencing were performed to produce largescale genomic analyses of these tumors.

This report demonstrates that ESCC and EAC are genomically distinct entities, as indicated by changes in regulatory 
pathways unique to each histologic subset. Whereas E-cadherin signaling and its regulatory pathways (ARF6 and FOXA) are increased in EACs, Wnt, syndecan, and p63 signaling are increased in ESCCs. Moreover, these latter changes are similar to what has been observed in squamous cell cancers of other primary sites, specifically the lung and oropharynx. ${ }^{2,3}$ Of note, human papilloma virus, which has been associated with SCCs at other sites, particularly the cervix and oropharynx, was not observed in esophageal SCC tumor specimens. These findings suggest that lineagespecific alterations can influence the progression of either EAC or ESCC. Further supporting the genetically distinct nature of EAC and ESCC, analysis of somatic genomic alterations identified clear differences in the patterns of gene copy number variations. Such copy number variations perturb cell-cycle regulation, cell signaling, and oncogenic amplification and have the potential to guide therapeutic intervention.

These findings not only highlight the differences between the 2 types of histology, but also identify strong associations between ESCC and EAC and tumors of similar histology at other primary sites. For example, DNA methylation analysis revealed that SCCs of the esophagus and oropharynx are more alike than ESCC and EAC, whereas integrative clustering analysis of the different platforms showed that EACs are indistinguishable from gastric cancers that express a high degree of chromosomal instability (CIN), similar to findings previously reported by our research group. ${ }^{4}$

These data validate the current criteria applied for staging esophageal cancer according to histology, and highlight how histology also should guide treatment options. In the forthcoming 8th edition of the American Joint Committee on Cancer/Union for International Cancer Control TNM staging criteria for esophageal cancer, tumor stage is further subclassified by grade based on histology and nodal status. Despite the different criteria applied for staging ESCC and EAC, esophageal cancer treatment does not account for tumor histology per se. In surgical trials, this may be due in part to the need to accrue adequate sample size when conducting comparative clinical trials, such as the Chemoradiotherapy for Oesophageal Cancer Followed by Surgery Study (CROSS) Group trial. That study compared the effects of preoperative chemoradiation to resection alone in patients with both ESCC and EAC. ${ }^{5}$ The results demonstrated a clear survival advantage from the addition of preoperative chemotherapy and radiation.

When subgroup analysis was performed, subjects treated for ESCC were more likely than those treated for EAC to have a complete pathological response ( $49 \%$ vs $23 \%$ ); however, histologic subtype was not correlated with a statistically significant difference in overall survival at 5 years. Longerterm analysis from the CROSS Group showed a median overall survival of 81.6 months in patients with ESCC, compared with 43.2 months for those with EAC. ${ }^{6}$ The large discrepancy in overall survival may be explained by the genomic differences between these histologic subtypes and differential response to platinum-based concurrent chemoradiation.

With respect to the anatomic and genetic similarity of cancers of the gastroesophageal junction, the Medical Research Council Adjuvant Gastric Infusional Chemotherapy (MAGIC) trial was conducted to compare perioperative chemotherapy and surgery to surgical therapy alone for patients with gastric cancer or cancer of the gastroesophageal junction. ${ }^{7}$ This trial demonstrated a statistically significant survival for patients receiving perioperative chemotherapy, with an overall survival of $36 \%$ at 5 years, superior to even the results reported initially by the CROSS Group. Major criticisms of this trial are the fact that the cohort included $25 \%$ of subjects with cancer of the lower esophagus or esophagogastric junction, as well as the high degree of toxicity of the chemotherapy regimen. Gastric cancer and EAC have long been thought to be 2 separate disease processes for which the same therapy could not be applied. Based on the results reported by TCGA, given the genomic homology of EAC and gastric cancer, the grouping of tumors in the MAGIC trial may be better suited than that of the CROSS trial, which combined EAC with ESCC.

These findings reported by TCGA demonstrate the importance of distinguishing between ESCC and EAC when considering treatment options. Expression profiles that show perturbations in various molecular pathways highlight potential differential responses to both targeted therapies and specific chemotherapeutic regimens. This recent report by the TCGA Research Network indicates that as thoracic oncologists we must proceed with caution in the design of future clinical trials, particularly regarding tumor histology, as we work to identify novel and more effective therapies for esophageal cancer, which remains a deadly disease, differences in histology notwithstanding.

\section{Conflict of Interest Statement}

Authors have nothing to disclose with regard to commercial support.

\section{References}

1. The Cancer Genome Atlas Research Network. Integrated genomic characterization of oesophageal carcinoma. Nature. 2017;541:169-75.

2. The Cancer Genome Atlas Research Network. Comprehensive genomic characterization of squamous cell lung cancers. Nature. 2012;489:519-25.

3. The Cancer Genome Atlas Research Network. Comprehensive genomic characterization of head and neck squamous cell carcinomas. Nature. 2015;517:576-82.

4. Ferrer-Torres D, Nancarrow DJ, Kuick R, Thomas DG, Nadal E, Lin J, et al Genomic similarity between gastroesophageal junction and esophageal Barrett's adenocarcinomas. Oncotarget. 2016;7:54867-82.

5. van Hagen $\mathrm{P}$, Hulshof $\mathrm{MC}$, van Lanschot JJ, Steyerburg EW, van Berge Henegouwen MI, Wijnhoven BP, et al. Preoperative chemoradiotherapy for esophageal or junctional cancer. $N$ Engl J Med. 2012;366:2074-84.

6. Shapiro J, van Lanschot JJ, Hulshof MC, van Hagen P, van Berge Henegouwen MI, Wijnhoven BP, et al. Neoadjuvant chemoradiotherapy plus surgery versus surgery alone for oesophageal or junctional cancer (CROSS): long-term results of a randomised controlled trial. Lancet Oncol. 2015;16:1090-8.

7. Cunningham D, Allum WH, Stenning SP, Thompson JN, Van de Velde CJ, Nicolson M, et al. MAGIC Trial Participants. Perioperative chemotherapy versus surgery alone for resectable gastroesophageal cancer. N Engl J Med. 2006;355:11-20. 